\title{
Índice de masa corporal materno pre-embarazo y riesgo de malformaciones congénitas específicas: evidencia de una relación dosis-respuesta
}

\author{
Block SR, Watkins SM, Salemi JL, Rutkowski R, Tanner JP, Correia JA, Kirby RS. \\ Maternal pre-pregnancy body mass index and risk of selected birth defects: evidence \\ of a dose-response relationship. Paediatr Perinat Epidemiol 2013;27(6):521-31. doi: \\ 10.1111/ppe.12084. \\ Análisis Crítico: Javiera Fuenzalida C. ${ }^{1}$, Claudio Vera P-G. ${ }^{2,3}$ MSc., Jorge Carvajal C. ${ }^{3}$ PhD. \\ ${ }^{1}$ Programa de Obstetricia y Ginecología, ${ }^{2}$ Unidad de Medicina Basada en Evidencia, ${ }^{3}$ Unidad de Medicina \\ Materno-Fetal. División de Obstetricia y Ginecología, Facultad de Medicina, Pontificia Universidad Católica de Chile.
}

\section{RESUMEN (1)}

Antecedentes: Este estudio investiga la relación entre el índice de masa corporal (IMC) previo al embarazo y 26 malformaciones al nacer identificadas en el Registro de Defectos de Nacimientos de Florida. Métodos: El IMC $(\mathrm{kg} / \mathrm{m} 2)$ previo al embarazo se categorizó en bajo peso $(<18,5)$, normopeso $(18,5$ $24,9)$, sobrepeso $(25-29,9)$ y obesidad $(\geq 30)$ entre las madres residentes de Florida sin diabetes pregestacional y que tuvieron partos de embarazos únicos entre marzo de 2004 y diciembre de 2009. La obesidad se clasificó en tres categorías: Obesidad I $(30,0-34,9)$, Obesidad II $(35,0-39,9)$ y Obesidad III $(\geq 40,0)$. Se utilizó regresión logística para calcular los Odds Ratios ajustados con un intervalo de confianza de $95 \%$, representando la asociación entre el IMC pre-embarazo y cada uno de los 26 defectos específicos al nacer y un compuesto de "cualquier malformación al nacer". Los modelos fueron ajustados según edad materna, raza/etnia, educación, tabaquismo, estado civil y origen. Resultados: En nacidos vivos la prevalencia de cualquier defecto al nacer aumenta con el incremento del IMC, de $3,9 \%$ en mujeres con bajo peso a $5,3 \%$ en mujeres con Obesidad III $(p<0,001)$. Los resultados muestran una relación directa dosis-respuesta entre el IMC materno pre-embarazo y 10 malformaciones en estudio (fisura palatina sin fisura labial, hernia diafragmática, hidrocefalia sin espina bífida, síndro- me de hipoplasia de ventrículo izquierdo, atresia y estenosis de válvula pulmonar, estenosis pilórica, estenosis y atresia rectal e intestinal, transposición de grandes arterias, tetralogía de Fallot y defectos septales ventriculares) y la categoría de "cualquier malformación al nacer". Al contrario, la gastrosquisis mostró una relación inversa y estadísticamente significativa con el IMC pre-embarazo. Conclusiones: Este estudio entrega evidencia del riesgo aumentado de malformaciones con el aumento de la obesidad pre-embarazo. Reducir la obesidad preembarazo, incluso entre las mujeres obesas, podría reducir la ocurrencia de estos defectos al nacer.

\section{ANÁLISIS DE LA INVESTIGACIÓN}

\section{A. Relevancia clínica de la investigación}

El aumento exponencial en la prevalencia de obesidad en la población (2) ha estimulado la búsqueda de eventuales asociaciones entre la obesidad previa al embarazo y el riesgo de resultados adversos perinatales $(3,4)$. En general, se han descrito efectos adversos relacionados con la mayor incidencia de diabetes gestacional, infertilidad, mortalidad perinatal y anomalías congénitas. Inicialmente, se describió el mayor riesgo de efectos adversos perinatales en relación a madres diabéticas pre-gestacionales o gestacionales (4-6). En cuanto al IMC previo al embarazo y su asociación 
con defectos al nacer, la evidencia de los estudios observacionales (10-16) y una revisión sistemática (12) es consistente en describir una asociación entre la obesidad materna y un mayor riesgo de malformaciones congénitas. La mayoría de los estudios se han focalizado en grupos heterogéneos de malformaciones, generalmente, agrupándolas por órgano por ejemplo: defectos cardíacos (7-9). Dado que las distintas malformaciones en cada órgano pueden tener diversas etiologías, resulta importante dividirlos por defecto específico. Por otro lado, una inconsistencia encontrada en las publicaciones previas tiene relación con la clasificación del peso materno, utilizando categorías diferentes que dificultan una comparación directa de los datos. La evidencia de una relación dosis-respuesta entre las distintas categorías de obesidad y malformaciones congénitas podría aportar a darle consistencia a la relación de causalidad. El objetivo de este estudio fue establecer y cuantificar la asociación entre anomalías congénitas específicas e IMC materno pre-embarazo obteniendo los datos de una base de datos amplia y extensa de un sistema de vigilancia con base poblacional.

\section{B. El estudio (1)}

Diseño: Es un estudio observacional caso control realizado de marzo de 2004 a diciembre de 2009 en la población de Florida, Estados Unidos. Población: Recién nacidos de embarazos únicos, hijos de madres residentes de Florida, sin diabetes pregestacional, identificados en el registro de malformaciones congénitas de Florida, con una o más malformaciones congénitas, excluyendo los casos con diagnóstico de trisomía 13,18 y 21 (casos). Comparación: Todos los niños nacidos vivos de madres residentes de Florida, sin malformaciones congénitas (no-casos). Evento de interés: relación entre malformaciones congénitas específicas e IMC materno pre-embarazo. Resultados: Se seleccionaron el $84 \%$ de los nacidos vivos elegibles en el periodo, luego de aplicar los criterios de selección y de excluir a las pacientes sin información de IMC. En la muestra, 5,5\% de las mujeres fueron clasificadas como de bajo peso, $52 \%$ de peso normal, $23,8 \%$ con sobrepeso y $18,7 \%$ con obesidad previa al embarazo. En el grupo de "casos", la prevalencia de obesidad fue mayor que en los "no casos" con un $21,6 \%$ y $18,6 \%$ respectivamente. La prevalencia de diabetes gestacional se relacionó con el IMC pre-gestacional y aumentó de $1,8 \%$ en mujeres de bajo peso, a $9,8 \%$ en mujeres con Obesidad III. EI grupo de "casos" presentó una mayor prevalencia de diabetes gestacional comparado con el de "no casos" con 5,0\% y 3,7\% respectivamente. Se observó que la asociación de "cualquier malformación" con el IMC pre-embarazo fue mayor a con IMC distintos de normopeso, y que aumentaba tanto con IMC bajo el normal como con IMC mayor al normal con una relación dosis respuesta (Tabla I). Las malformaciones específicas de hidrocefalia, espina bífida y defectos ventriculares septales tuvieron mayor asociación con bajo peso. Una serie de malformaciones congénitas que incluyó: fisura labial con o sin fisura palatina, fisura palatina sin fisura labial, hernia diafragmática, defectos de cojinetes endocárdicos, obstrucción urinaria baja, estenosis/atresia válvulas pulmonares, estenosis pilórica, estenosis/atresia rectal o intestinal, espina bífida sin anencefalia, transposición de grandes arterias, tetralogía de Fallot y defecto ventricular septal, presentaron mayor asociación con obesidad comparado con peso normal. Por otro lado, se observó un menor riesgo de tener un recién nacido con gastrosquisis en las pacientes con sobrepeso y

Tabla I

MALFORMACIONES CONGÉNITAS Y CONDICIÓN NUTRICIONAL PREEMBARAZO

\begin{tabular}{lccc}
\hline $\begin{array}{l}\text { Condición } \\
\text { nutricional preembarazo }\end{array}$ & $\begin{array}{c}\text { Casos } \\
\mathrm{n}=44.624 \\
\%\end{array}$ & $\begin{array}{c}\text { Controles } \\
\mathrm{n}=1.079 .746 \\
\%\end{array}$ & $\begin{array}{c}\text { OR (IC 95\%) } \\
\text { ajustada } f \text {. Cualquier } \\
\text { malformación congénita }\end{array}$ \\
\hline Bajo Peso & 5,50 & 5,46 & $1,08(1,04$ a 1,13) \\
Peso normal & 49,04 & 52,09 & 1 \\
Sobrepeso & 23,98 & 23,83 & $1,05(1,03$ a 1,08) \\
Obesidad I & $11,98^{*}$ & $11,04^{*}$ & $1,12(1,09$ a 1,16) \\
Obesidad II & $5,65^{*}$ & $4,64^{*}$ & $1,26(1,21$ a 1,31) \\
Obesidad III & $3,90^{*}$ & $2,9^{*}$ & $1,37(1,31$ a 1,47) \\
\hline
\end{tabular}

*Estimados a partir de la Figura 1 y Tablas 1, 3 y 4. $f$ Regresión logística ajustada por raza/etnia materna, educación materna, tabaco materno, estado marital materno, lugar de nacimiento materno y edad materna. 
obesidad. Cuando se utilizó el factor de corrección de Bonferroni con $\mathrm{p} \leq 0,0004$ para múltiples comparaciones, se encontró que de los 19 hallazgos estadísticamente significativos, solo 6 permanecían significativos luego de la corrección, sin embargo, no están claramente identificados en las tablas o texto. Se observaron cinco cardiopatías congénitas con asociaciones crecientes a medida que aumenta con el grado de obesidad; al aplicar el factor de corrección Bonferroni $p \leq 0,0018$, solo cuatro de las asociaciones permanecieron estadísticamente significativas, "cualquier malformación congénita", estenosis/atresia de válvula pulmonar, tetralogía de Fallot y defecto septal ventricular.

\section{Análisis crítico}

Validez interna: Los autores no describen el diseño, dada la pregunta principal se clasificó como estudio observacional caso y control. En este tipo de diseño el riesgo de sesgo de información es difícil de dimensionar puesto que la información fue extraída de una base de datos que depende de los datos obtenidos de múltiples fuentes como certificados de nacimiento, certificados de defunción, documentos de hospitalización o alta; luego pueden haber errores como pérdida de información, información errada o clasificaciones inadecuadas. Además, no se registran en esta base de datos los óbitos o abortos que también podrían haber sido causados por una malformación congénita y no se consideraron diversos factores confundentes en el análisis como el consumo de drogas, la exposición a tóxicos, o el trabajo de las pacientes. Para la evaluación de la exposición incluye información del IMC pre-embarazo registrado en el momento del nacimiento con riesgo de subestimación. Tanto casos y controles tienen aparentemente una similar oportunidad de exposición a los diferentes estratos de IMC, no se incluyen las estimaciones crudas de la asociación, los autores ajustaron por factores confundentes disponibles e incluyeron un factor de corrección de la significancia, tomando en cuenta las múltiples comparaciones. En el análisis llama la atención que la asociación está expresada tomando como base a la categoría de peso normal en vez de expresar la asociación en términos de las Odds Ratio de cada una de las categorías de IMC dado el estado de caso o control. Validez externa: El estudio incluye un amplio período de evaluación, incluyendo el $84 \%$ de la población elegible del período, con una prevalencia de malformaciones congénitas de $3,9 \%$. Excluye las madres con diabetes gestacional disminuyendo el impacto de éste factor confundente, si bien reconocen la limitación de que algún porcentaje de las pacientes con diabetes gestacional podrían ser pregestacionales. El estudio representa a la población de Florida, que incluye población de diversos orígenes étnicos en proporciones distintas a las de nuestro medio. Además, del total de pacientes excluidas por falta de datos maternos, un $44 \%$ correspondía a aquellas mujeres de origen no americano que podrían ser el reflejo de nuestra población. Finalmente, si analizamos la distribución de peso de la población chilena, observamos mayor prevalencia tanto de sobrepeso como de obesidad comparado con este estudio. En resumen, es un estudio cuyos resultados son consistentes con lo previamente publicado en la literatura, en el cual se explora una relación de temporalidad entre el factor de riesgo y el resultado final evaluado, y se evidencia una relación dosis-respuesta coherente. Conclusión: Se trata de un estudio observacional caso control con un número adecuado de pacientes para evaluar malformaciones específicas y un alto riesgo de sesgo que muestra una débil asociación entre el riesgo de malformaciones congénitas y el IMC pre-embarazo de mujeres de una población de Florida y además muestra una relación dosisrespuesta de las malformaciones en general y de algunas malformaciones específicas. Este estudio apoya la asociación entre malformaciones congénitas y obesidad pre-embarazo haciendo plausible la hipótesis de que una apropiada nutrición previa al embarazo puede aportar a la diminución de malformaciones congénitas asociadas a esta causa.

\section{REFERENCIAS}

1. Block SR, Watkins SM, Salemi JL, Rutkowski R, Tanner JP, Correia JA, Kirby RS. Maternal pre-pregnancy body mass index and risk of selected birth defects: evidence of a dose-response relationship. Paediatr Perinat Epidemiol 2013;27:521-31. doi: 10.1111/ppe.12084.

2. Zera C, McGirr S, Oken E. Screening for obesity in reproductive-aged women. Preventing Chronic Disease [serial on the internet] 2011;8(6):A 125.

3. Siega-Riz AM, Siega-Riz AM, Laraia B. The implications of maternal overweight and obesity on the course of pregnancy and birth outcomes. Matern Child Health J 2006;10(5 Suppl):S153-6.

4. Aberg A, Westbom L. Association between maternal pre-existing or gestational diabetes and health problems in children. Acta Paediatrica 2001;90:746-50.

5. Aberg A, Westbom L, Kallen B. Congenital malformations among infants whose mothers had gestational diabetes or preexisting diabetes. Early Hum Develop 2001;61:85-95.

6. Moore LL, Singer MR, Bradlee ML, Rothman KJ, Milunsky A. A prospective study of the risk of congenital defects associated with maternal obesity and diabetes mellitus. Epidemiology 2000;11:689-94.

7. Gilboa SM, Correa A, Botto LD, Rasmussen SA, Waller DK, Hobbs CA, et al. Association between prepregnancy body mass index and congenital heart defects. Am J Obstet Gynecol 2010;202:51.e1-51.e10.

8. Cedergren MI, Kallen BA. Maternal obesity and infant heart defects. Obes Res 2003;11:1065-71. 
9. Mills JL, Troendle J, Conley MR, Carter T, Druschel CM. Maternal obesity and congenital heart defects: a population-based study. Am J Clin Nutr 2010;91:15439.

10. Khalil HS, Saleh AM, Subhani SN. Maternal obesity and neonatal congenital cardiovascular defects. Inter J Gynaecol Obstet 2008;102:232-6.

11. Blomberg MI, Kallen B. Maternal obesity and morbid obesity: the risk for birth defects in the offspring. Birth Defects Res A Clin Mol Teratol 2010;88:35-40.

12. Stothard KJ, Tennant PW, Bell R, Rankin J. Maternal overweight and obesity and the risk of congenital anomalies: a systematic review and meta-analysis. JAMA 2009;301:636-50.

13. Shaw GM, Todoroff K, Schaffer DM, Selvin S. Maternal height and prepregnancy body mass index as risk factors for selected congenital anomalies. Paediatr Perinat Epidemiol 2000;14:234-9.

14. Waller DK, Shaw GM, Rasmussen SA, Hobbs CA, Canfield MA, Siega-Riz AM, et al. Prepregnancy obesity as a risk factor for structural birth defects. Arch Pediatr Adolesc Med 2007;161:745-50.

15. Marengo L, Farag NH, Canfield M. Body mass index and birth defects: Texas, 2005-2008. Matern Child Health J 2013. Disponible en: http://download. springer.com/static/pdf/791/art\%253A10.1007\%252 Fs10995-012-1214-5.pdf?auth66=1384455709_6e19 1e287c6cd1f797010df7acf7c621\&ext=.pdf

16. Watkins ML, Rasmussen SA, Honein MA, Botto LD, Moore CA. Maternal obesity and risk for birth defects. Pediatrics 2003;111:1152-8. 